\title{
Workspace Narrative Exploration: Overcoming Interruption-Caused Context Loss in Information Seeking Tasks*
}

\author{
Youngjoo Park and Richard Furuta \\ Center for the Study of Digital Libraries \\ and Department of Computer Science and Engineering \\ Texas A\&M University \\ College Station, TX 77843, USA \\ \{yjoo9317, furuta\}@cse.tamu .edu
}

\begin{abstract}
As digital libraries become more prevalent and as the amount of accessible online information grows, users often must consult diverse information collections in carrying out tasks. Simultaneously, the impact of frequent interruptions on task performance gets more severe. To manage the negative effects of interruptions on work performance, workers often engage in task management activities to ensure they are better prepared to resume suspended tasks. However, managing tasks causes additional cognitive burden and incurs a time cost to users who already are experiencing demands on their attention and time. We describe a system that allows users to browse their previous workspace intuitively, and enhances continuity of their tasks by supporting them to retrieve desired work context more quickly and easily.
\end{abstract}

Keywords: Context browser, narrative, task, contextual cue, discontinuity, user interfaces.

\section{Introduction}

The diversity and volume of online information are growing daily. As a result, tasks performed by information workers often include various information pieces carefully selected from many different collections on the World-Wide Web or on huge local hard disk drives. For information workers, performing tasks typically coincides with frequent interruptions and resumptions, which leads to the need for an efficient mitigating interface for minimizing negative effects of interruptions. In particular, a significant task, such as a problem solving task, tends to be complex and to last a longer period of time, making the task and the users vulnerable to interruption [1]. When an interruption occurs, the relationship between the task and a set of associated information resources easily can be broken as the task's environment-i.e., desktop status - starts being adjusted to the new task's requirements; e.g., as users close windows and open new ones. Unfortunately, the current computing environment lacks a service by which users may seamlessly 1) restore the context when the task was suspended and 2) resume the unfinished task with rich contextual artifacts.

\footnotetext{
* This material is based upon work supported by the National Science Foundation under Grant No. IIS-0534314.
} 


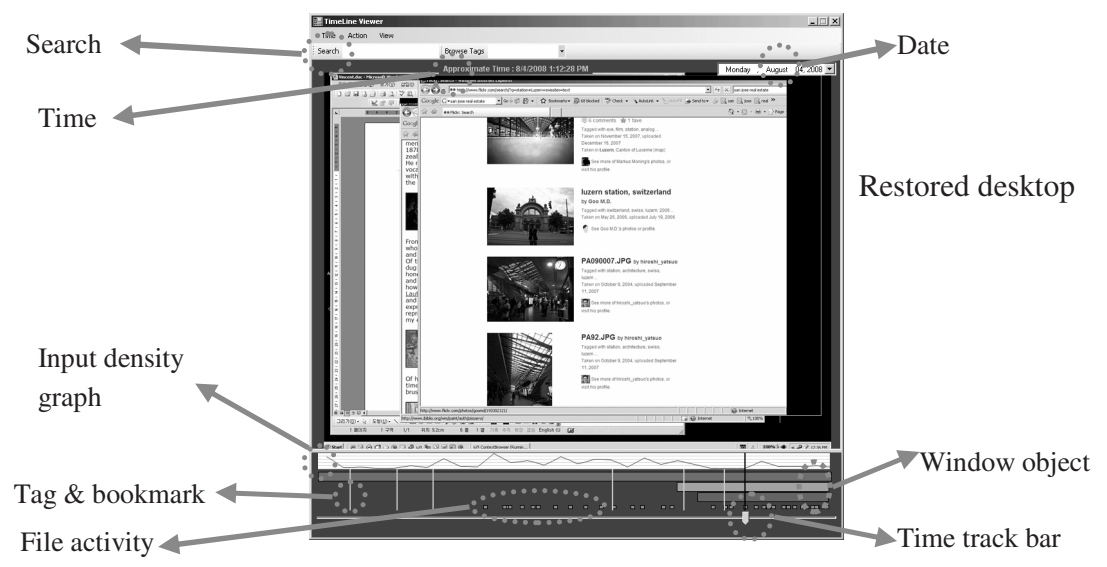

Fig. 1. Timeline interface of a context browser

In this paper, we briefly present an interface for users' workspace narrative exploration, Context Browser, and describe a study in which subjects performed multiple tasks and encountered various interruptions in a more realistic working environment. The work reported here extends our previous study [2], which showed that the subjects with the context browser were able to prepare for task resumption more quickly and retrieve more associated information than the subjects without the context browser.

\section{Context Browser: Keeping Workspace Narrative Close}

A user's desktop fluidly accommodates various work settings; a workspace narrative is a stream of a user's activities spread across various work settings, i.e., a contextual archive. We hypothesized that digitizing workspace narratives would enable the novel opportunity to index, restore, reuse, manipulate, explore and, most importantly, search users' cognitive and task contexts in which associated windows were visible. This is easier for users than is requiring explicit user intervention to save discrete information at a specific moment, which is typical in existing task management tools.

The Context Browser implements the idea of placing a user into an appropriate context to carry out a task with less cognitive and time costs. Its timeline viewer is the primary interface that users use to explore narratives of a desktop to find their desired context or any necessary information resources; see Figure 1. A reconstructed desktop in the timeline view interface supports typical desktop interactions with a mouse such as window selection and dragging, and even double-clicking to launch a window. The interface also supports launching the whole desktop status to retrieve a desired work context. The system is equipped with 1) a tag and bookmark mechanism to allow future revisiting and reuse and 2) a search engine as well that utilizes a vector space model to measure cosine similarities between a query and a metadata element in the contextual archive, such as window titles, URLs, Web page titles and process names. 


\section{User Study and Findings}

24 subjects ( 5 female), with ages ranging from 20 to 39 , participated in the study. They were not compensated. 6 out of the 14 married subjects had a child(ren). To perform between subject measures, we divided subjects evenly into two separate groups. Subjects in group 1 used the context browser and those in group 2 did not.

To make a typical office environment with frequent interruptions and multiple tasks, we prepared a set of three tasks and assigned them to subjects prior to the study. The study was conducted in two separate sessions; each taking approximately one hour. The period between the two sessions was about $24 \sim 48$ hours.

Task \#1 asked the subjects to find pictures of given 9 cities (5 pictures a city). From now on, we refer to this task as the City pictures task.

Task \#2, the Van Gogh task, asked them to search for Van Gogh's famous paintings with their metadata, i.e., location, title, year, and save them in the document.

In Task \#3, subjects planned a move to San Jose, CA, due to a job offer with a monthly salary of $\$ 5,000$ after taxes. They needed to seek information on housing or apartments and to find at least 3 candidates to move in. We refer to this task as the San Jose task.

\subsection{Recall and Precision}

The City pictures task echoes such tasks that are associated with a considerable amount of information resources, such as graphs and images. In reality, there might be the situation in which users need to retrieve and review all resources they had already seen previously either to verify that they have a proper result or modify the current result. To simulate this situation, in the $2^{\text {nd }}$ session we additionally asked subjects to do the following - "Given 10 minutes, find the images that you believe that you saved while performing this task."

Group 1 (avg=0.643, sd=0.03) showed better recall rate than group 2 (avg=0.4, $\mathrm{sd}=0.14), \mathrm{t}=5.45, \mathrm{df}=22, \mathrm{p}<0.0001$. Particularly, the difference in precision is more obvious than the one in recall. By having the subjects in group 1 exposed to narratives of prior desktop status, group $1(\mathrm{avg}=0.933$, $\mathrm{sd}=0.46)$ collected more images with much higher precision within a limited period than group $2(\mathrm{avg}=0.59, \mathrm{sd}=0.15) \mathrm{did}$, $\mathrm{t}=7.23, \mathrm{df}=22, \mathrm{p}<0.0001$.

\subsection{Time Lag to Recover Search Contexts}

The San Jose task forced subjects to execute cognitively loaded activities, i.e., repeated search activities, to narrow down their search list to a manageable size. They had to resolve various parameters, such as a choice between house and apartment, a number of bedrooms, prices, regions and so forth. In the $2^{\text {nd }}$ session, we asked them to look for two more houses or apartments sharing similar specification to the one they liked most previously. In terms of a time cost to restore a desired search list, group 1 $(\operatorname{avg}=245$ sec., $s d=70)$ successfully retrieved the context faster than group $2(a v g=521$ sec., $\mathrm{sd}=225), \mathrm{t}=-4.06, \mathrm{df}=22, \mathrm{p}<0.001$. The subjects in group 1 found the context browser useful to get them back to the state where they were evaluating houses or 
apartments on the list, which was the fruit of various search activities. However, the subjects in group 2 again had to go through all the steps that they took previously to find them, which sometimes painfully cost a long period to execute the given task.

\subsection{Performance After a Lengthy Period}

We wondered if the Context Browser would continue to be useful after some time had passed, especially for the subjects in group 2, who had not used it previously. We thus conducted one additional experiment with re-invited 6 subjects from group 2 six weeks after the previous study. We let them use the context browser at this time to see if there was any improvement in handling the problems that they did previously after more than a month delay. During this additional session, we again assigned the same protocols, i.e., finding the images that you saved previously and a similar house or apartment, and measured as we did 6 weeks earlier. We performed a paired-samples $\mathrm{T}$ test comparing these results with their previous performance and discovered that with the context browser they were able to retrieve more images, i.e., a better recall, (mean difference $=-0.17, \mathrm{t}=-6.7, \mathrm{df}=5, \mathrm{p}<0.001)$ and the images more correctly, i.e., a better precision, (mean difference $=-0.218, \mathrm{t}=-8.26, \mathrm{df}=5, \mathrm{p}<0.001$ ) even after the 6 week delay. They also easily found the house or apartment they liked and successfully searched similar places less stressfully and more quickly (mean difference $=177.16$ sec., $\mathrm{t}=3.47, \mathrm{df}=5, \mathrm{p}=0.018$ ). This finding also indicates that by only browsing the timeline, subjects were able to fetch their desired information and contexts since the archives for group 2 were not arranged except by time.

\section{Conclusion}

With the Context Browser, the study showed that users are better able to remember and recognize semantic information presented by the system than with a typical desktop environment. This is because the workspace narratives successfully captured semantically meaningful contextual cues and the context browser provided users with services, such as timeline browsing, tag and bookmark, search, and so forth, by which they can easily browse and retrieve appropriate work settings. In addition, the subjects expressed that they found the context browser very useful since it handled a tedious information organization task and kept their desired information within a close distance asking less both time and cognitive costs to find it.

\section{References}

1. Czerwinski, M., Horvitz, E., Wilhite, S.: A diary study of task switching and interruptions. In: Proceedings of CHI, pp. 175-182 (2004)

2. Park, Y., Furuta, R.: Keeping narratives of a desktop to enhance continuity of on-going tasks. In: Proceedings of JCDL, pp. 393-396 (2008) 